\title{
Double sine-Gordon model revisited
}

\author{
G. Takács*and F. Wágner ${ }^{\dagger}$ \\ Theoretical Physics Research Group, Hungarian Academy of Sciences \\ H-1117 Budapest, Pázmány Péter sétány 1/A
}

1st November 2018

\begin{abstract}
We reconsider the mass spectrum of double sine-Gordon theory where recent semiclassical results called into question the previously accepted picture. We use the Truncated Conformal Space Approach (TCSA) to investigate the claims. We demonstrate that the numerics supports the original results, and strongly disagrees with those obtained from semiclassical soliton form factor techniques. Besides the numerical analysis, we also discuss the underlying theoretical arguments.
\end{abstract}

\section{Introduction}

Double sine-Gordon theory has attracted interest recently chiefly because it is a prototype of non-integrable field theory which can be understood by application of techniques developed in the context of integrable field theories [1]. It has several interesting applications such as to the study of massive Schwinger model (two-dimensional quantum electrodynamics) and a generalized Ashkin-Teller model (a quantum spin system) which are discussed in [1]. Another application to the one-dimensional Hubbard model is examined in [2] (together with the generalized Ashkin-Teller model mentioned above). A further potentially interesting application of the two-(and multi-)frequency sine-Gordon model is for ultra-short optical pulses propagating in resonant degenerate medium [3].

In an earlier work the authors (and coworkers) studied this model using non-perturbative finite size techniques and established the phase diagram of the model [4] (which was recently extended to the multi-frequency generalization [5]). In particular we used form factor perturbation theory introduced by Delfino and Mussardo in [1] to predict mass spectra and verify them using the truncated conformal space approach (TCSA) [6].

\footnotetext{
*Email: takacs@elte.hu

${ }^{\dagger}$ E-mail: wferi@niif.hu
} 
Recently Mussardo et al. [7] applied a semiclassical soliton form factor technique developed by Goldstone and Jackiw [8] to study the mass spectrum of the theory. They obtained results that contradict explicitely some of the results obtained using form factor perturbation theory in [4]. In principle, the truncated conformal space approach applied in [4] could decide the question, but the accuracy achieved there is not sufficient for this purpose.

The present work reports a systematic study of this issue using improved TCSA numerics which do have the required accuracy. Besides the numerical comparisons we also address the theoretical arguments raised in [7] to support their case. We find a convincing and consistent picture, both numerically and theoretically, which allows us to clarify the issue.

We would like to stress that the problem at hand is much more than a simple numerical discrepancy: it is the validity of the form factor perturbation theory and that of semiclassical techniques that is at stake here, both of which are very promising tools for non-perturbative investigation of non-integrable quantum field theories.

We start with recalling some necessary facts about the double sine-Gordon model to set the stage in Section 2. Section 3 describes the mass spectrum obtained from form factor perturbation theory in [4] on the one hand and from the semiclassical soliton form factor technique on the other [7]. Section 4 describes the numerical work and the results obtained, while Section 5 is devoted to the underlying theoretical issues. We conclude briefly in Section 6.

\section{The double sine-Gordon model}

The classical model is defined by the Lagrangian density

$$
\mathcal{L}_{\text {classical }}=\frac{1}{2} \partial_{\mu} \varphi \partial^{\mu} \varphi-\mu_{0} \cos \beta \varphi-\lambda_{0} \cos \left(\frac{\beta}{2} \varphi+\delta\right)
$$

For $\lambda_{0}=0$ we recover standard sine-Gordon theory, where the spectrum is known to consist of a soliton doublet of mass

$$
M_{0}=\frac{8 \sqrt{\mu_{0}}}{\beta}
$$

and a continuum of breathers

$$
m=2 M_{0} \sin \frac{\pi}{2} \alpha \quad, \quad 0<\alpha<1
$$

We define the quantum version of the model using perturbed conformal field theory as in [4]. The Hamiltonian can be written

$$
H_{\mathrm{PCFT}}=H_{\mathrm{c}=1}+\mu \int d x: \cos \beta \varphi:+\lambda \int d x: \cos \left(\frac{\beta}{2} \varphi+\delta\right):
$$


where

$$
H_{\mathrm{c}=1}=\int d x: \frac{1}{2}\left(\partial_{t} \varphi\right)^{2}+\frac{1}{2}\left(\partial_{x} \varphi\right)^{2}:
$$

is the Hamiltonian of a $c=1$ free boson CFT. The quantum spectrum for $\lambda=0$ consists of a soliton doublet of mass $M$ which is related to the coupling $\mu$ by [9]

$$
\mu=\frac{2 \Gamma(\Delta)}{\pi \Gamma(1-\Delta)}\left(\frac{\sqrt{\pi} \Gamma\left(\frac{1}{2-2 \Delta}\right) M}{2 \Gamma\left(\frac{\Delta}{2-2 \Delta}\right)}\right)^{2-2 \Delta} \quad, \quad \Delta=\frac{\beta^{2}}{8 \pi}
$$

which satisfies the semiclassical asymptotics

$$
\mu \sim \frac{\beta^{2}}{64} M^{2} \quad, \quad \beta \rightarrow 0
$$

which means that $\mu$ can be identified with $\mu_{0}$ in that limit (similar results hold for $\lambda$ and $\left.\lambda_{0}\right)$. In the quantum theory, the breather spectrum is discrete and their numbers depend on the frequency $\beta$ :

$$
m_{n}=2 M \sin \frac{\pi}{2} \frac{n \beta^{2}}{8 \pi-\beta^{2}} \quad, \quad n=1, \ldots,\left[\frac{8 \pi}{\beta^{2}}\right]-1
$$

Double sine-Gordon model can be considered as a non-integrable perturbation of the integrable sine-Gordon field theory [1]. Because of the presence of the second cosine term, the field must be taken to have the periodicity

$$
\varphi \equiv \varphi+\frac{4 \pi}{\beta}
$$

which is called a 2-folded model following [10]. Therefore at $\lambda=0$ the theory has two degenerate vacua corresponding to the classical solutions

$$
\varphi=0 \quad \text { and } \quad \frac{2 \pi}{\beta}
$$

As a result, each breather comes in two copies, and soliton states must be labeled with the vacua between which they mediate, thereby implementing a so-called 'kink' structure [10]. In this paper we treat $\lambda$ as the non-integrable perturbing coupling constant, although it is equally possible to swap the roles of the cosine terms in this respect.

The most interesting case is $\delta=\frac{\pi}{2}$ when for small $\lambda$ the two vacua remain degenerate until a critical value is reached where the theory undergoes a phase transition. This was shown at the classical level (mean field theory) by [1] to be of second order in the Ising universality class, and it was established that this holds even after taking quantum fluctuations into account (non-perturbatively) in [4]. This is the model in which we are the most interested, although we shall also use the $\delta=0$ case as a testing ground for our numerical calculations. 


\section{Mass spectrum predictions}

\subsection{Form factor perturbation theory}

Form factor perturbation theory (FFPT) was introduced in [11] to provide a way of expanding physical quantities in the non-integrable perturbing coupling. It was applied to the double sine-Gordon model in [4]. For our present purposes, we only need the mass shift of the first breather, which reads ${ }^{1}$

$$
\delta m_{1}^{(k)}=\frac{\lambda \mathcal{G}_{\beta / 2}(\beta) \mathcal{N}}{M} \cos (\pi(k-1)+\delta) \exp \left\{-\frac{1}{\pi} \int_{0}^{\pi p} d t \frac{t}{\sin t}\right\}
$$

where $k=1,2$ labels the two copies of the first breather, $p=\beta^{2} /\left(8 \pi-\beta^{2}\right)$,

$$
\begin{aligned}
\mathcal{G}_{a}(\beta) & =\left[\frac{M \sqrt{\pi} \Gamma\left(\frac{4 \pi}{8 \pi-\beta^{2}}\right)}{2 \Gamma\left(\frac{\beta^{2} / 2}{8 \pi-\beta^{2}}\right)}\right]^{\frac{a^{2}}{4 \pi}} \\
& \times \exp \left\{\int_{0}^{\infty} \frac{d t}{t}\left[\frac{\sinh ^{2}\left(\frac{a \beta}{4 \pi} t\right)}{2 \sinh \left(\frac{\beta^{2}}{8 \pi} t\right) \sinh (t) \cosh \left(\left(1-\frac{\beta^{2}}{8 \pi}\right) t\right)}-\frac{a^{2}}{4 \pi} e^{-2 t}\right]\right\}
\end{aligned}
$$

and

$$
\mathcal{N}=\exp \left\{4 \int \frac{d t}{t} \frac{\sinh (t) \sinh (p t) \sinh ((1+p) t)}{\sinh ^{2} 2 t}\right\}
$$

It is an important prediction of FFPT that the mass corrections vanish when $\delta=\frac{\pi}{2}$ and that also

$$
\delta m^{(1)}=-\delta m^{(2)}
$$

in general.

\subsection{Masses from semiclassical soliton form factors}

In [7] a semiclassical method, based on the Goldstone-Jackiw semiclassical kink form factor [8], was used to determine the spectrum of double sine-Gordon theory. Here we give a brief summary of their results for the case $\delta=\frac{\pi}{2}$. They claim that the perturbation by $\cos \left(\frac{\beta}{2} \varphi+\frac{\pi}{2}\right)$ induces a linear mass correction to the mass of the $n$th breather, and obtain the following result:

$$
m_{n}^{(L, S)}=m_{n} \pm 2 \pi \frac{\lambda_{0} \beta}{\sqrt{\mu_{0}}}\left(\frac{1}{\beta^{2}} \sin \left(n \frac{\beta^{2}}{16}\right)-\frac{n}{16} \cos \left(n \frac{\beta^{2}}{16}\right)\right)+O\left(\lambda_{0}^{2}\right)
$$

where $L$ and $S$ index the splitting up of the $n$th breather level into what they call a 'short' and a 'long' breather.

\footnotetext{
${ }^{1}$ We corrected some misprints in the published version of [4].
} 
The main problem and the central issue in this paper, is that this disagrees with the FFPT results in the previous subsection that give a vanishing linear correction to the breather masses, and it also disagrees with the numerical studies in [4] where the masses of the two copies of any breather levels remained degenerate as long as $\lambda$ was lower than the critical value, where a second order phase transition induced a mass gap between the ground states and also lifted the degeneracy of the breather masses. However, the numerical studies in [4] were not aimed at studying the breather levels and the data produced for that work, while still accessible, have insufficient precision to decide whether a splitting of the order of (3.4) is actually present. In addition, the authors of [7] presented some arguments to support the result (3.4), to which we shall return later. For the moment the issue is to decide which of the predictions is borne out by the dynamics of the model.

Using the soliton mass $M_{0}=8 \sqrt{\mu_{0}} / \beta$, the mass correction can be written as

$$
\frac{\delta m_{n}^{(L, S)}}{M_{0}}= \pm 16 \pi \frac{\lambda_{0}}{M_{0}^{2}}\left(\frac{1}{\beta^{2}} \sin \left(n \frac{\beta^{2}}{16}\right)-\frac{n}{16} \cos \left(n \frac{\beta^{2}}{16}\right)\right)=\frac{\pi n^{3} \beta^{4}}{768} \frac{\lambda_{0}}{M_{0}^{2}}+O\left(\beta^{6}\right)
$$

In terms of the dimensionless coupling constant

$$
\chi_{0}=\frac{\lambda_{0}}{M_{0}^{2}}
$$

this can be written

$$
\frac{\delta m_{n}^{(L, S)}}{M_{0}}= \pm \frac{\pi n^{3} \beta^{4}}{768} \chi_{0}+O\left(\beta^{6}\right)
$$

where we again absorbed the difference between the classical and quantum mass/coupling relations into the higher order correction term.

The dimensionless coupling of the quantum theory is defined by

$$
\chi=\frac{\lambda}{M^{2-\frac{\beta^{2}}{16 \pi^{2}}}}
$$

(where the difference in the exponent of $M$ is just the anomalous dimension of the perturbing operator). In the semiclassical limit $\chi$ only differs from $\chi_{0}$ by terms higher order in $\beta^{2}$ as discussed in the previous section, and similarly for $M$ and $M_{0}$. Therefore we can still use formula (3.5) to leading order in $\beta$ in the quantum theory just replacing $M_{0}$ by $M$ and $\chi_{0}$ by $\chi$

$$
\frac{\delta m_{n}^{(L, S)}}{M}= \pm \frac{\pi n^{3} \beta^{4}}{768} \chi+O\left(\beta^{6}\right)
$$

For our purposes we are only interested in the first breather $(n=1)$ and the comparisons to numerical results will be done at the following couplings:

$$
\begin{array}{ll}
\beta=\frac{\sqrt{4 \pi}}{2.5} \quad: \quad \frac{\delta m_{1}^{(L, S)}}{M}= \pm 0.0165367 \chi+\ldots \\
\beta=\frac{\sqrt{4 \pi}}{2.2} \quad: \quad \frac{\delta m_{1}^{(L, S)}}{M}= \pm 0.0275751 \chi+\ldots
\end{array}
$$




\section{TCSA analysis}

We now aim to get the mass spectrum solving the model directly using the so-called truncated conformal space approach (TCSA) introduced by Yurov and Zamolodchikov in [6]. It was extended to perturbations of $c=1$ free boson models in [12] and applied to the double sine-Gordon model in [4].

The application of TCSA to perturbations of $c=1$ free boson CFT is described in detail in [4] and references therein, therefore we do not go into the details here. We mention only that this method gives the finite volume spectrum of the theory, from which the masses can be extracted by locating the so-called scaling region in which the mass gap function (the energy difference between the one-particle state and the corresponding vacuum state) is approximately constant. More precisely, we locate the volume range where the magnitude of the numerical derivative of the mass gap function is smallest and define the mass as the value the mass gap function takes there. The Hilbert space can be split up into sectors differing in the value of the winding number of the field $\varphi$ and the eigenvalue of total spatial momentum, and the mass gap functions we are interested in can be measured from the sector with no winding and zero momentum.

We improved on the precision of the TCSA significantly with respect to [4]. First, we chose smaller values for $\beta$ which makes TCSA converge faster in the models at hand. This is also advantageous since it means we are closer to the classical limit and so we expect to be able to test the semiclassical prediction (3.4). Second, we pushed the cutoff much higher using more machine power, and also by applying the parity symmetries to project the Hilbert space into even and odd subspaces, using that the TCSA Hamiltonian is block diagonal in the appropriate basis. This made possible treating more than 18000 states in reasonable computing time, and allowed us to achieve the accuracy which makes it possible to judge the correctness of the semiclassical prediction (3.4).

\section{1 $\delta=0$ : testing the numerics}

In this case the model has a symmetry

$$
\varphi \rightarrow-\varphi
$$

which can be used to project the TCSA Hilbert space onto an even and an odd sector. This reduces the number of states and helps to achieve higher values of the cutoff. In order to be on safe ground, the program was run both with and without a projection for several values of $\beta, \chi$ and the cutoff. The resulting spectra were always identical (within the precision of floating point arithmetics), independently of the values of the parameters and of the cutoff, which confirms that the symmetry projection was correctly implemented.

We also compared the $\chi=0$ to predictions of exact $S$ matrix theory, which they matched perfectly within the truncation errors. The vacua can be found in the even, while the one-particle states in the odd sector. They can be matched against each other using the fact that the bulk energy constant of the two vacua are different, therefore the two vacua (and also the two corresponding one-particle states) have a different slope in the scaling 
region. The redefinition $\lambda \rightarrow-\lambda$ leaves the total spectrum invariant (in both the odd and the even sector separately), but exchanges the role of the two sets of states, therefore it is expected that

$$
m_{1}^{(1,2)}(-\lambda)=m_{1}^{(2,1)}(\lambda)
$$

We choose the following value for the coupling

$$
\beta=\frac{\sqrt{4 \pi}}{2.5}=1.4179631 \ldots
$$

For mass determination, the cutoff was placed at level 18 resulting in 9334 states in the even and 9319 states in the odd sector respectively.

At this value of $\beta$ there are

$$
\left[\frac{8 \pi}{\beta^{2}}\right]-1=10
$$

breathers in the $\lambda=0$ spectrum which shows that this model is deeply in the semiclassical regime. The FFPT formula (3.1) gives the following prediction for the mass corrections:

$$
\frac{\delta m_{1}^{(1,2)}}{M}= \pm 0.95392713 \ldots \times \chi
$$

We summarize the measured values of the masses in table 4.1 and plotted them against predictions of form factor perturbation theory in figure 4.1. It is obvious that the measured masses follow well the predictions from form factor perturbation theory, but there are deviations that grow with the coupling. These are expected to be higher order effects. However, second order corrections should cancel from the difference, since sending $\lambda$ to $-\lambda$ exchanges the identity of the two particles [4]. In fact, the mass differences agree with form factor perturbation theory within the estimated TCSA errors which can be estimated from cut-off dependence of the energy levels and the flatness of the gap function in the scaling region. We can also fit the coefficient of (4.2) against the mass splitting measured in TCSA, for which we find

$$
0.95441 \pm 0.00032
$$

The central value differs by only 0.0005 from (4.2), consistent with the $1 \sigma$ error range of the fit.

It is also interesting to examine the mass correction in the classical limit. Calculating the curvature of the field potential around its minima at $\varphi=0$ and $\varphi=\frac{2 \pi}{\beta}$ gives

$$
\frac{\delta m_{1}^{(1,2)}}{M_{0}}= \pm \chi_{0} \quad, \quad \chi_{0}=\frac{\lambda_{0}}{M_{0}^{2}}
$$

We can see that quantum corrections to the coefficient are of the order 0.05, and the TCSA method is able to measure them with one percent accuracy. It is also useful to remark that the semiclassical expansion of quantum corrections to mass ratios goes in the parameter

$$
\frac{\beta^{2}}{8 \pi}
$$




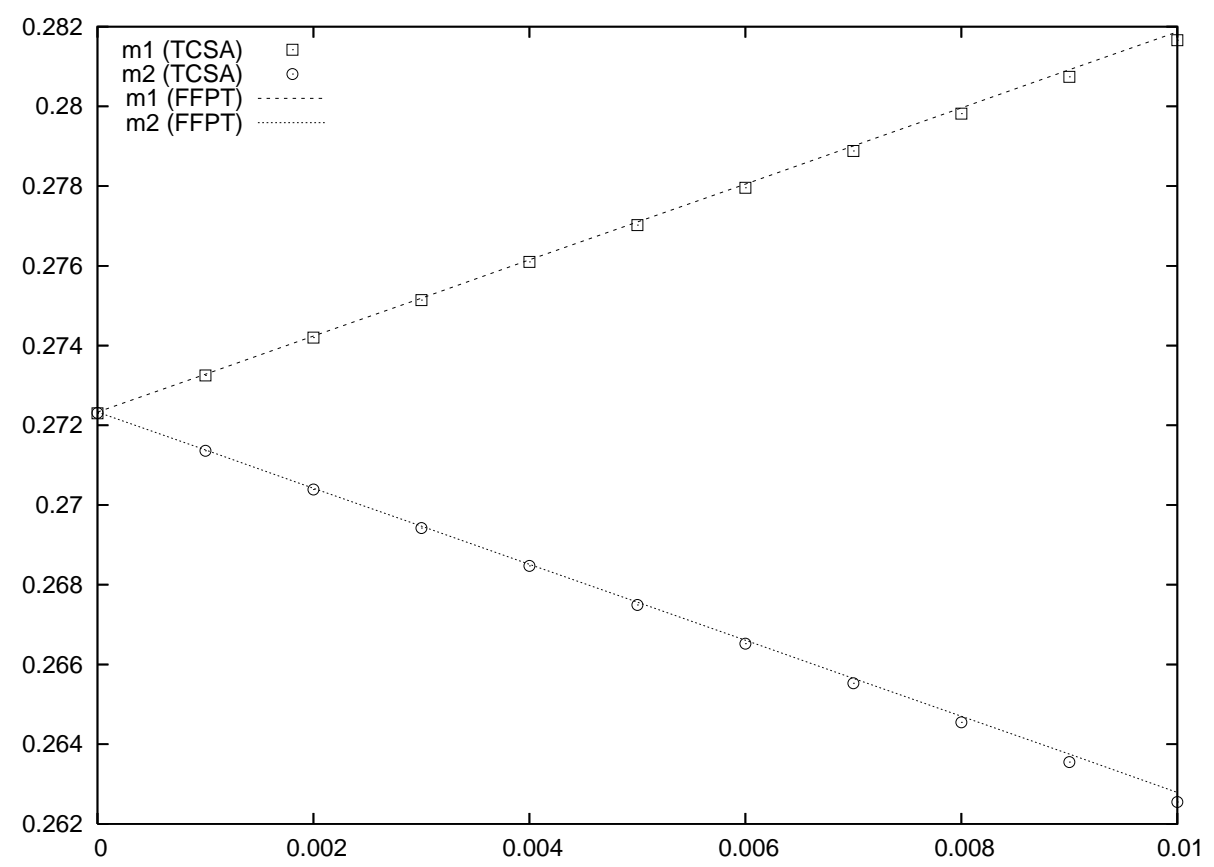

(a) The two masses, denoted here by $\mathrm{m} 1$ and $\mathrm{m} 2$, from TCSA and FFPT

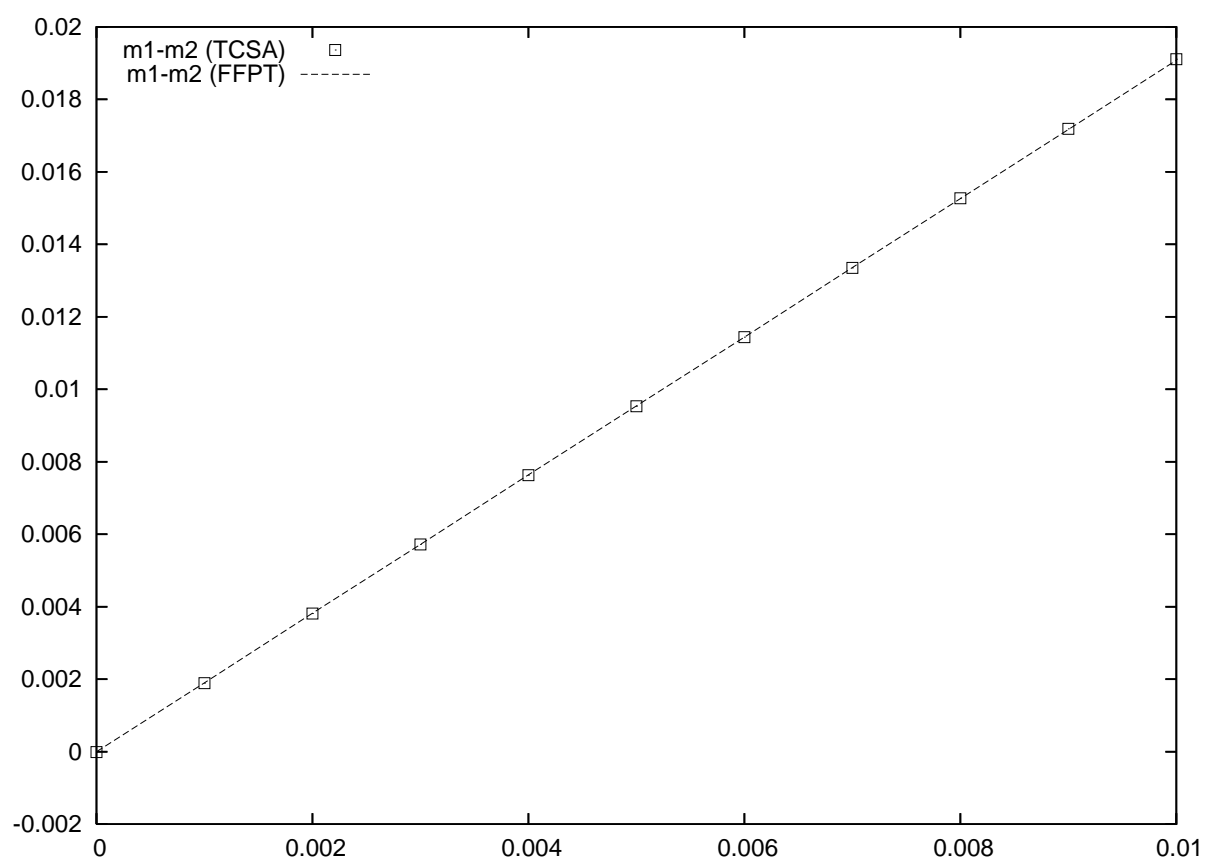

(b) The mass splitting from TCSA and from FFPT

Figure 4.1: Comparing masses measured from TCSA and predicted using FFPT for $\beta=$ $\frac{\sqrt{4 \pi}}{2.5}, \delta=0$. All data are in units of the unperturbed soliton mass $M . m 1$ and $m 2$ denote the two masses $m_{1}^{(1,2)}$, respectively. The error bars of TCSA data are too small to visualize. 


\begin{tabular}{|c|c|c|c|c|c|c|}
\hline$\chi$ & $\begin{array}{c}m_{1}^{(1)} \\
(\mathrm{TCSA})\end{array}$ & $\begin{array}{c}m_{1}^{(2)} \\
(\mathrm{TCSA})\end{array}$ & $\begin{array}{c}m_{1}^{(1)} \\
(\mathrm{FFPT})\end{array}$ & $\begin{array}{c}m_{1}^{(2)} \\
(\mathrm{FFPT})\end{array}$ & $\begin{array}{c}m_{1}^{(1)}-m_{1}^{(2)} \\
(\mathrm{TCSA})\end{array}$ & $\begin{array}{c}m_{1}^{(1)}-m_{1}^{(2)} \\
(\mathrm{FFPT})\end{array}$ \\
\hline \hline 0 & 0.27230 & 0.27231 & 0.27233 & 0.27233 & -0.00001 & 0 \\
\hline 0.001 & 0.27325 & 0.27136 & 0.27328 & 0.27138 & 0.00019 & 0.00020 \\
\hline 0.002 & 0.27420 & 0.27039 & 0.27424 & 0.27042 & 0.00381 & 0.00380 \\
\hline 0.003 & 0.27514 & 0.26942 & 0.27519 & 0.26947 & 0.00572 & 0.00572 \\
\hline 0.004 & 0.27610 & 0.26847 & 0.27615 & 0.26851 & 0.00763 & 0.00764 \\
\hline 0.005 & 0.27702 & 0.26749 & 0.27710 & 0.26756 & 0.00953 & 0.00954 \\
\hline 0.006 & 0.27796 & 0.26652 & 0.27805 & 0.26661 & 0.01144 & 0.01144 \\
\hline 0.007 & 0.27888 & 0.26553 & 0.27901 & 0.26565 & 0.01335 & 0.01336 \\
\hline 0.008 & 0.27982 & 0.26455 & 0.27996 & 0.26470 & 0.01527 & 0.01526 \\
\hline
\end{tabular}

Table 4.1: Comparing masses measured from TCSA and predicted using FFPT for $\beta=$ $\frac{\sqrt{4 \pi}}{2.5}, \delta=0$. All data are in units of the unperturbed soliton mass $M$. TCSA mass measurements have an estimated error of order \pm 0.00002 .

(as can be seen e.g. by expanding the unperturbed sine-Gordon breather/soliton mass ratio (2.1) for small $\beta$ ) and for this particular value of $\beta$ we have

$$
\frac{\beta^{2}}{8 \pi}=0.08
$$

which explains why the corrections to the classical limit are so small.

\section{2 $\delta=\pi / 2$ : the acid test}

In this case there is a symmetry

$$
\varphi \rightarrow \frac{2 \pi}{\beta}-\varphi
$$

which we used for projection. We again performed runs with and without a projection to confirm that the program was functioning correctly. In this case, both the even and the odd sector contains a vacuum and a one-particle state.

For the value of $\beta$ used in the previous section, we obtain the masses listed in table (4.2). At the same truncation level (18) the dimension of the even sector is 9334 while that of the odd is 9319 as before, but the state vectors assigned to these sectors are different since now we use (4.4) instead of (4.1) for the projection. In the unperturbed sine-Gordon model $(\lambda=0)$ both projections can be done and the spectra compared to check the validity of the program, and we found a perfect match within the precision of floating point arithmetics.

The spectrum does not change if we change the sign of $\chi$ in either sectors, so the masses $m_{1}^{ \pm}$are even functions of $\lambda$. This fact alone already precludes the existence of the linear correction (3.6), but we shall go through a detailed analysis in order to take into account the numerical uncertainties of the TCSA mass determination as well. We can also plot the 


\begin{tabular}{|c|c|c|c|c|}
\hline$\chi$ & $m_{1}^{+}$ & $m_{1}^{-}$ & $m_{1}^{+}-m_{1}^{-}$(TCSA) & $m_{1}^{+}-m_{1}^{-}(3.6)$ \\
\hline \hline 0 & 0.27230 & 0.27231 & -0.00001 & 0 \\
\hline \pm 0.010 & 0.27154 & 0.27154 & 0 & 0.00033 \\
\hline \pm 0.015 & 0.27059 & 0.27057 & 0.00002 & 0.00050 \\
\hline \pm 0.020 & 0.26925 & 0.26925 & 0 & 0.00066 \\
\hline \pm 0.025 & 0.26750 & 0.26746 & -0.00004 & 0.00083 \\
\hline \pm 0.030 & 0.26536 & 0.26530 & 0.00006 & 0.00099 \\
\hline \pm 0.035 & 0.26276 & 0.26274 & 0.00002 & 0.00116 \\
\hline \pm 0.040 & 0.25975 & 0.25971 & 0.00004 & 0.00132 \\
\hline \pm 0.045 & 0.25625 & 0.25624 & 0.00001 & 0.00149 \\
\hline \pm 0.050 & 0.25236 & 0.25229 & 0.00007 & 0.00165 \\
\hline
\end{tabular}

Table 4.2: Masses measured from TCSA for $\beta=\frac{\sqrt{4 \pi}}{2.5}, \delta=\frac{\pi}{2}$ in units of the unperturbed soliton mass $M$. TCSA mass measurements have an estimated error of \pm 0.00002 . We also list the mass splittings measured from TCSA and compare it to the predictions (3.6).

measured mass difference and the prediction from (3.6) as in figure 4.2, and the mismatch is obvious from the plot.

We can also fit a function of the form

$$
m_{1}^{ \pm}=m_{1}+a_{ \pm} \chi+b_{ \pm} \chi^{2}+c_{ \pm} \chi^{3}+d_{ \pm} \chi^{4}
$$

to the measured masses in the $\chi \in[-0.05 \ldots 0.05]$ range. The fit results are

$$
\begin{array}{ll}
a_{+}=0 \pm 0.00010 & a_{-}=0 \pm 0.000054 \\
b_{+}=-7.606 \pm 0.018 & b_{-}=-7.625 \pm 0.011 \\
c_{+}=0 \pm 0.054 & c_{-}=0 \pm 0.033 \\
d_{+}=-152.4 \pm 6.9 & d_{-}=-149.5 \pm 4.4
\end{array}
$$

This must be compared to the prediction (3.6) which gives $a_{ \pm}= \pm 0.0165367$. We see that this value is two orders of magnitude larger than the uncertainty of the fit. Since the model in question is very deeply in the semiclassical value, even higher order quantum corrections cannot come to the rescue (we have seen in the previous section that they are at the level of a few percent). The conclusion is that the prediction (3.6) is not valid to the order $\beta^{4}$ which makes the conclusions of [7] based on these results unfounded (an analysis of the theoretical arguments in that paper is also given in the following section).

We also analyzed the mass spectrum for $\beta=\frac{\sqrt{4 \pi}}{2.2}$. This model has 8 breathers, so it is also very much in the semiclassical regime $\left(\beta^{2} / 8 \pi=0.10331 \ldots\right)$. Comparing FFPT with classical mass corrections again gives an estimate for quantum corrections at the few percent level. However, higher $\beta$ means that the accuracy of TCSA is necessarily somewhat lower, but is still enough for our purposes. At truncation level 18, we found 7963 states in the even and 7978 states in the odd sector. The measured masses are summarized in table 4.3 , and the mass splitting is plotted in figure 4.3. 


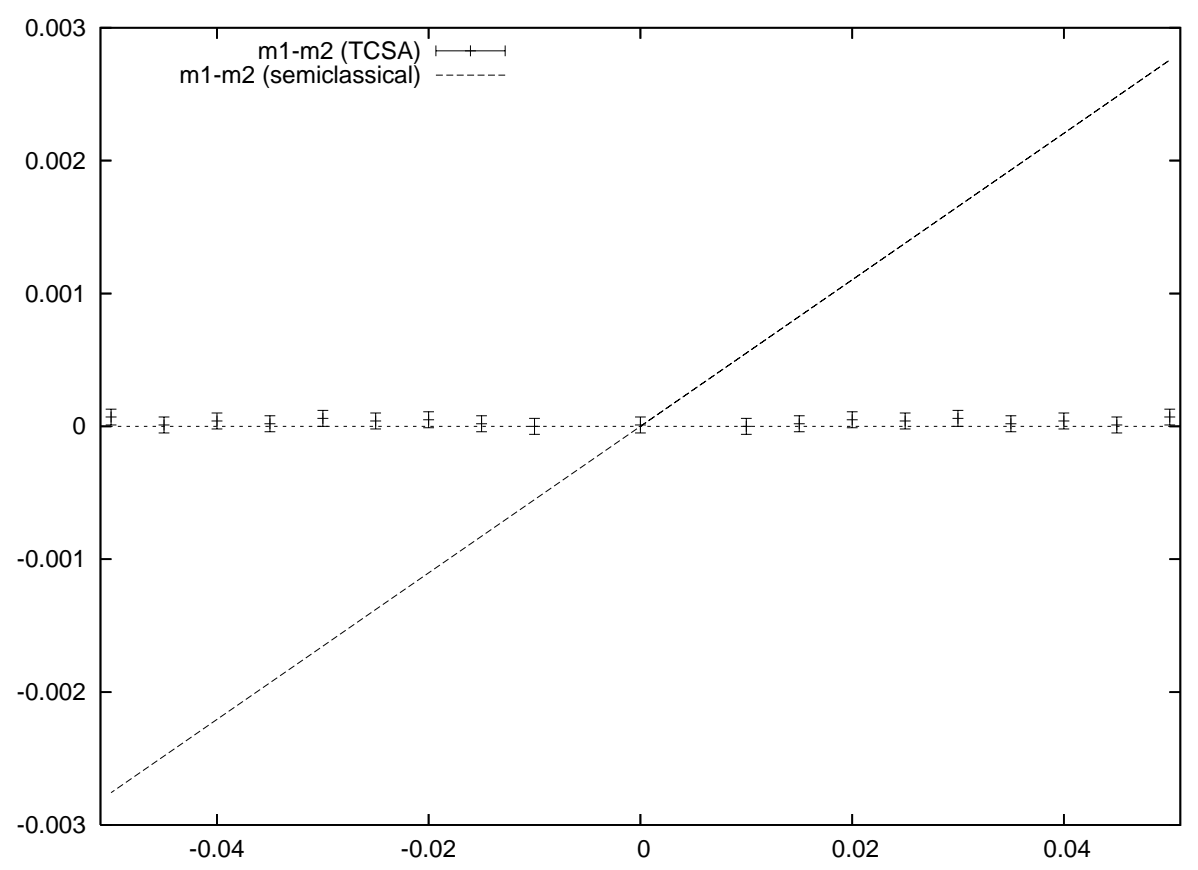

Figure 4.2: The mass splitting measured from TCSA compared to the semiclassical prediction (3.6) at $\beta=\frac{\sqrt{4 \pi}}{2.5}, \delta=\frac{\pi}{2}$. The error bars indicate the estimated magnitude of truncation errors.

\begin{tabular}{|c|c|c|c|c|}
\hline$\chi$ & $m_{1}^{+}$ & $m_{1}^{-}$ & $m_{1}^{+}-m_{1}^{-}$(TCSA) & $m_{1}^{+}-m_{1}^{-}(3.6)$ \\
\hline \hline 0 & 0.36002 & 0.35986 & 0.00016 & 0 \\
\hline 0.010 & 0.35940 & 0.35927 & 0.00013 & 0.00055 \\
\hline 0.015 & 0.35864 & 0.35853 & 0.00011 & 0.00083 \\
\hline 0.020 & 0.35758 & 0.35747 & 0.00011 & 0.00110 \\
\hline 0.025 & 0.35622 & 0.35611 & 0.00011 & 0.00137 \\
\hline 0.030 & 0.35449 & 0.35444 & 0.00005 & 0.00165 \\
\hline 0.035 & 0.35248 & 0.35242 & 0.00006 & 0.00193 \\
\hline 0.040 & 0.35011 & 0.35011 & 0 & 0.00220 \\
\hline 0.045 & 0.34747 & 0.34751 & -0.00004 & 0.00248 \\
\hline 0.050 & 0.34443 & 0.34445 & -0.00002 & 0.00276 \\
\hline
\end{tabular}

Table 4.3: Masses measured from TCSA for $\beta=\frac{\sqrt{4 \pi}}{2.2}, \delta=\frac{\pi}{2}$ in units of the unperturbed soliton mass $M$. TCSA mass measurements have an estimated error of order \pm 0.00005 . We also list the mass splittings measured from TCSA and compare it to the predictions (3.6). 


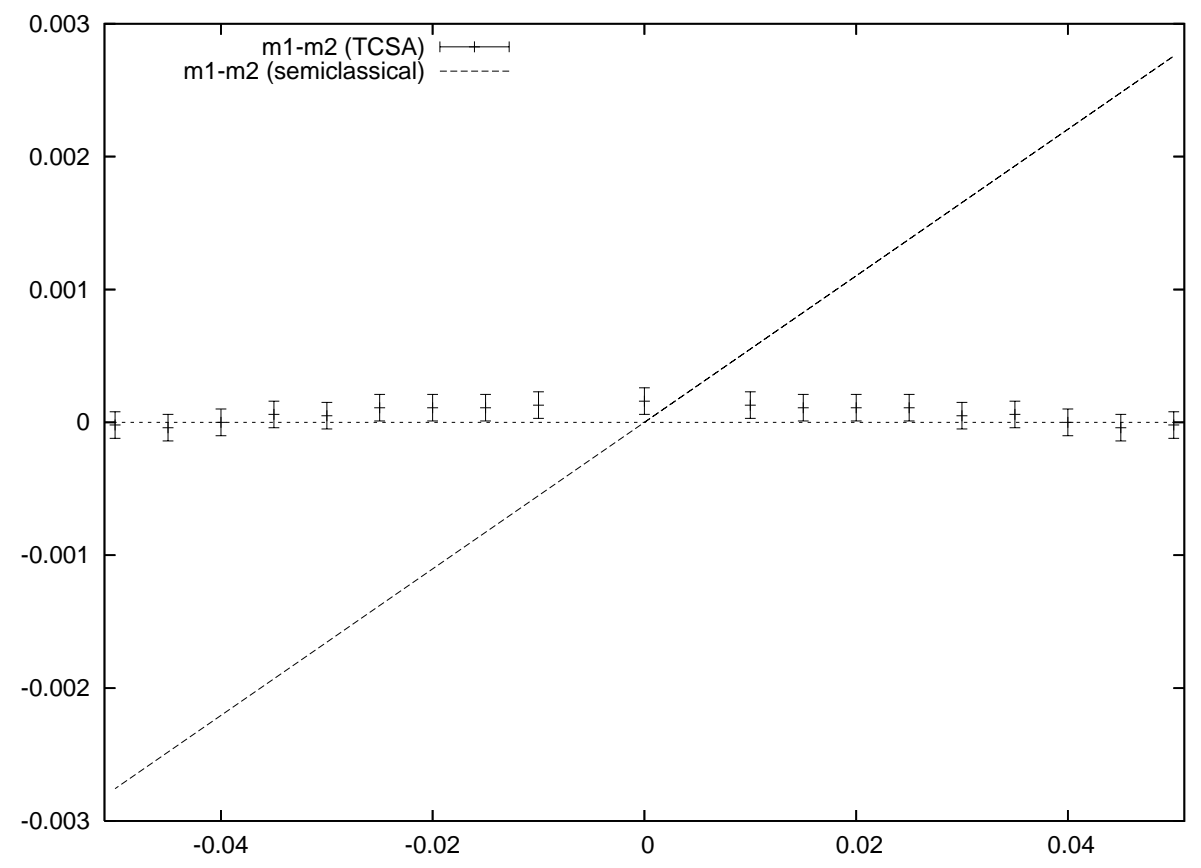

Figure 4.3: The mass splitting measured from TCSA compared to the semiclassical prediction (3.6) at $\beta=\frac{\sqrt{4 \pi}}{2.5}, \delta=\frac{\pi}{2}$. The error bars indicate the estimated magnitude of truncation errors. 
We can again perform a fit in the range $\chi \in[-0.05 \ldots 0.05]$ with the results

$$
\begin{array}{ll}
a_{+}=0 \pm 0.00008 & a_{-}=0 \pm 0.0002 \\
b_{+}=-6.076 \pm 0.017 & b_{-}=-5.966 \pm 0.021 \\
c_{+}=0 \pm 0.053 & c_{-}=0 \pm 0.11 \\
d_{+}=-63.3 \pm 6.5 & d_{-}=-77.3 \pm 8.6
\end{array}
$$

The prediction (3.6) gives

$$
a_{ \pm}= \pm 0.0275751
$$

which is again ruled out by two orders of magnitude.

\section{$5 \quad$ Theoretical arguments}

In the previous section we demonstrated that TCSA data are strongly incompatible with the semiclassical analysis of the mass spectrum in [7], while they are in full accordance with the analysis of our earlier paper [4] based on form factor perturbation theory.

\subsection{Issues concerning the semiclassical form factor method}

Considering that the prediction (3.4) is only semiclassical, one may hope that higher order corrections can adjust it to be compatible to the numerical data. This hope is unfounded, however, since we have seen that quantum corrections can be estimated to be at most a few percent at the values of $\beta$ we considered. Therefore we must conclude that (3.4) is not correct even to its lowest order.

This is not surprising in the light of the fact that the leading order term (3.5) is of order $\beta^{4}$. The semiclassical computation presented in [7] is simply not valid to this order. Quantum corrections to soliton form factor and soliton mass should be taken into account before extracting the breather mass from the pole of the form factor. To order $\beta^{2}$, however, (3.5) is in accord with form factor perturbation theory that predicts no linear correction to the masses.

We would also like to discuss briefly the mass corrections predicted in [7] for the case $\delta=0$. They obtain

$$
\delta m_{n}^{( \pm)}= \pm \frac{\beta \lambda_{0}}{8 \sqrt{\mu_{0}}}\left[\left(1-\log \frac{\lambda_{0}}{16 \mu_{0}}\right) \frac{32}{\beta^{2}} \sin \left(n \frac{\beta^{2}}{32}\right)+n \log \frac{\lambda_{0}}{16 \mu_{0}} \cos \left(n \frac{\beta^{2}}{32}\right)\right]+O\left(\lambda_{0}^{2}\right)
$$

Expanding in $\beta$

$$
\frac{\delta m_{n}^{ \pm}}{M_{0}}= \pm\left(n+\beta^{4} \frac{n^{3}}{6144}\left(1-\log \frac{\lambda_{0}^{2}}{256 \mu_{0}^{2}}\right)+O\left(\beta^{6}\right)\right) \frac{\lambda_{0}}{M_{0}^{2}}+O\left(\lambda_{0}^{2}\right)
$$

The classical limit

$$
\frac{\delta m_{n}^{ \pm}}{M_{0}}= \pm n \frac{\lambda_{0}}{M_{0}^{2}}+O\left(\lambda_{0}^{2}\right)
$$


is correctly reproduced by this formula. However, the $\beta^{4}$ term cannot be trusted because of the same reasons as above.

Expansion of the FFPT mass correction (4.2) produces terms of all orders in $\beta^{2}$. It is obvious that a $\beta^{2}$ term is also generated from (5.1) when we replace the classical parameters $\lambda_{0}$ and $M_{0}$ by the PCFT coupling $\lambda$ and the quantum soliton mass $M$ since the coupling and the mass are redefined at each order in the $\beta^{2}$ expansion. For this reason alone the terms in (5.1) that are higher order in $\beta$ cannot be compared directly to (4.2).

However, there is a deeper problem with (5.1): the presence of the nonanalytic (logarithmic) dependence in $\lambda_{0}$ would suggest that although the masses have the correct limit when $\lambda_{0} \rightarrow 0$, the $\lambda_{0} \neq 0$ case cannot be treated as a perturbation of the $\lambda_{0}=0$ model.

In contrast, both conformal perturbation theory and form factor perturbation theory shows a rather different behaviour. Conformal perturbation theory produces a series expansion in $\lambda$ and $\mu$, which contains no ultraviolet divergences at any order if $\beta^{2}<4 \pi$. There does not seem to be any way to produce nonanalytic behaviour in the coupling $\lambda$ in the regime of small $\beta$ : even a partial resummation over $\mu$ would leave us with an analytic expansion in powers of $\lambda$ despite the fact that it may produce nonanalytic terms in $\mu$ (due to infrared divergences present in the perturbation around the conformal field theory describing the massless ultraviolet fixed point). This partial resummation, on the other hand, is just expected to be identical to form factor perturbation theory (which is free of the infrared divergences since it expands around a massive field theory) which gives a first order correction in $\lambda$ with no $\log \lambda$ terms, and matches very well with the numerics of TCSA as shown in subsection 3.1. On the other hand, since the semiclassical soliton form factor calculation in [7] is not self-consistent to order $\beta^{4}$, it cannot be used to argue for the presence of $\log \lambda$ corrections. Before attempting any comparison to TCSA results, one must compute all the relevant loop corrections and suitably renormalize $\lambda_{0}, M_{0}$ to $\lambda, M$, which is out of the scope of the present work.

We remark that even if all of the above is carried out, it could prove very difficult to provide any conclusive evidence against the existence of $\lambda \log \lambda$ corrections of order $\beta^{4}$, since these cannot be separated easily from the already present $\lambda$ corrections, which are of order $\beta^{0}$. However, the agreement of the measured mass splitting (illustrated in figure $4.1(\mathrm{~b})$ ) with the FFPT result (4.2) is very suggestive: the linear coefficient can be determined to a relative accuracy of $5 \times 10^{-4}$, and we still have not exhausted all resources to make the TCSA even more accurate: increasing the cut level is still a definite possibility (although it increases the required computer time very fast, with around the 3.2th power of the dimension of the Hilbert space), and extrapolating the cutoff dependence of measured masses to infinite cutoff is another one.

\section{2 $\quad$ FFPT and neutral states in the $\delta=\frac{\pi}{2}$ case}

In the paper [7] the authors also produce a scenario in the $\delta=\frac{\pi}{2}$ case to support the presence of the linear mass correction term (3.4). Let us recall the main line of their argument.

It is known that the breathers arise as bound states in the soliton-antisoliton $(s \bar{s})$ 
channel, with alternating $C$-parity. Namely, the $n$th breather $B_{n}$ with $n$ odd/even arises as a bound state in a channel where the $s \bar{s}$ wave-function is odd/even under charge conjugation (interchanging soliton and antisoliton), respectively.

The authors argue that such a situation is very unique to the sine-Gordon model and holds only if the non-integrable coupling $\lambda$ is not present. They claim that there exists another breather state of "wrong" $C$-parity and the $n$ breather is actually a doublet $B_{n}^{ \pm}$ (the upper index denoting $C$-parity), but the states of wrong parity $B_{n}^{-(-1)^{n}}$ decouple from the model which means that the corresponding three-particle couplings have the behaviour

$$
f_{s \bar{s}}^{B_{n}^{+}}=0 \text { for } n \text { odd } f_{s \bar{s}}^{B_{n}^{-}}=0 \text { for } n \text { even }
$$

so that only one pole appears in the $s \bar{s}$ channel for any $n$, with alternating $C$-parity, since the other residue vanishes, and therefore the $2 \times 2 s \bar{s}$ scattering matrix degenerates to a one-dimensional projection at each pole.

They continue by demonstrating that a modified form factor perturbation theory, adjusted to take into account this degeneracy produces a linear correction to the breather mass of equal magnitude and opposite sign to the two breather copies, thus making the picture appear consistent with their semiclassical result (3.4). For $k$-folded models where the periodicity of the field is determined so that vacua are only identified after $k$ periods

$$
\varphi \equiv \varphi+k \frac{2 \pi}{\beta}
$$

they predict $2 k$ copies of each breather, twice as much as the $k$-folded model has, half of which is claimed to decouple from the model.

There are, however, several problems with this scenario. Extensive investigation of the spectrum of sine-Gordon theory has been carried out using the TCSA approach. No sign of these extra states was ever detected, although the Hilbert space was guaranteed to be complete by taking all states of the ultraviolet conformal field theory into account (truncated by an upper energy cut, but all the experience with TCSA shows that this cannot be the reason for missing these states). Varying $\beta$ down from the free fermion value $\sqrt{4 \pi}$ one can observe directly how the breather states appear. The result can be stated very simply in the case of the 1 -folded model. We can identify the $s \bar{s}$ states in the spectrum by fitting their finite size energy dependence using Bethe Ansatz and the exact $S$-matrix [13], and since the $S$-matrix eigenvalue in the odd/even parity channel is different, we can even identify the parity of the states. When $\beta$ crosses the threshold corresponding to the appearance of the $n$ breather, a single $s \bar{s}$ state crosses below the $2 M$ limit (where $M$ is the soliton mass), and this is a state of $C$-parity $(-1)^{n}$ : new breather states appear one by one, and always with the right parity. There is no trace of the extra states, and this carries over to the $k$-folded case as well: at each threshold value of $\beta$ we always observe $k$ copies of the appropriate breathers appearing in the spectrum (cf. [10] for some examples of spectra; we have produced many of them for different values of $\beta$, of which the published paper naturally contains only a few representative examples.)

In the above argument, there is one possible loophole: TCSA uses periodic boundary conditions, and it may be argued that these exclude some states from the spectrum or 
produce some identification between states, leaving only half of the breather states that are actually there in infinite volume ${ }^{2}$. However, even in this case one expects the mass spectrum extracted from TCSA to display the correct dependence on the couplings, and so the fact that our numerical results present such a strong case against any linear term (or, in fact, any odd power) in $\lambda$ remains a very compelling evidence against this scenario.

The paper [7] also brings up an analogy with the theory $\operatorname{RSO}_{3}$ (the factorized scattering theory of the tricritical Ising model). The corresponding $S$-matrix has no bound state poles, but such poles can be introduced by dressing with appropriate CDD factors. $R S O S_{3}$ has three vacua which can be illustrated by the potential

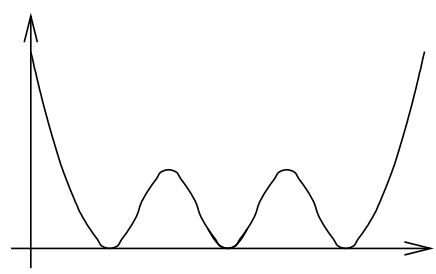

The middle well of these is flanked by two others, and a simple calculation of the bootstrap spectrum reveals two breather bound states over it. These can be described as bound states of a kink going from the middle vacuum to one side and of a kink coming the way back. This seems to be analogous to the sine-Gordon vacuum structure, which is periodically infinite, but any chosen vacuum has again two neighbours.

The analogy is flawed, however, because in sine-Gordon theory due to the periodic vacuum structure the boson is actually compactified on a circle. In the standard (1-folded) case, the identification is

$$
\varphi \equiv \varphi+\frac{2 \pi}{\beta}
$$

and therefore all vacua are identified. It does not make much sense to talk about two vacua flanking a middle one as in the $R S O S_{3}$ case where all three vacua are physically distinct and show up as 3 different energy levels in a finite volume (e.g. TCSA) spectrum, which become exponentially degenerate in the large volume limit. The 1-folded sine-Gordon model, on the other hand, has a unique ground state even in the large volume limit. In the $k$-folded model there are $k$ different vacua (with the topology of a circle), which is also manifest in the finite volume spectrum [10]. This vacuum structure is corroborated both by TCSA studies and by the exact description of the finite volume spectrum using the Destri-de Vega nonlinear integral equation (NLIE) [14] (see also $[12,13,10]$ ). It accounts readily for the $k$ copies of each breather, but there is no additional doubling in the spectrum: the physics of these vacua is rather different from that of the $\mathrm{RSO}_{3}$ case.

Our final conclusion is that the arguments given by the authors of [7] for these additional states are not well-founded, while the numerical data show that the dynamics of the model

${ }^{2}$ This issue was raised by G. Mussardo in a private discussion. There are known examples where the TCSA spectrum does not include all states of the infinite volume theory because of the selectivity of boundary conditions, however they are not very useful analogies of double sine-Gordon theory, similarly to the case of $\mathrm{RSOS}_{3}$ discussed in the main text. 
can be described very well by the usual form factor perturbation theory applied in [4] without any need for adjustments.

\section{Conclusions}

The numerical analysis has shown with quite large precision that the conclusions of [7] regarding the mass spectrum are untenable, more precisely the linear mass corrections at

$\delta=\frac{\pi}{2}$ predicted by them are ruled out by two orders of magnitude. The main issue seems to be that they trust the results from the semiclassical form factor approach to an order at which it is simply not consistent without performing the appropriate loop corrections as well. We also considered their theoretical arguments and have shown that they do not stand up to closer scrutiny.

In contrast, the numerics is fully consistent with our original conclusions about the mass spectrum in [4]. Obviously, this must not be taken as a complete verification of these results. It is always possible that there are effects that the numerics misses because of its finite precision and otherwise limited scope but which may be relevant for theoretical understanding. For the present we can only say that under closer examination the picture presented in [4] holds up. That picture is also consistent with many theoretical expectations and accumulated experience from the long study of two-dimensional quantum field theory (the 'folklore'), but certainly this is not a water-tight proof either.

We conclude by noting that the double sine-Gordon model is an interesting quantum field theory in its own right, and we hope that continued work will shed more light on its workings.

\section{Acknowledgments}

G.T. would like to thank G. Mussardo, Z. Bajnok and L. Palla for discussions. This work was partially supported by the EC network "EUCLID", contract number HPRN-CT-200200325, and Hungarian research funds OTKA D42209, T037674 and T043582. G.T. was also supported by a Bolyai János Research Fellowship.

\section{References}

[1] G. Delfino and G. Mussardo, Nucl. Phys. B516 (1998) 675-703, hep-th/9709028.

[2] M. Fabrizio, A.O. Gogolin and A.A. Nersesyan, Nucl. Phys. B580 (2000) 647-687, cond-mat/0001227.

[3] R.K. Bullough, P.J. Caudry and H.M. Gibbs, in Solitons, Eds. R.K. Bullough and P.J. Caudry, Topics in Current Physics v. 17, Springer-Verlag, 1980.

[4] Z. Bajnok, L.Palla, G. Takács and F. Wágner, Nucl. Phys. B601 (2001) 503-538, hep-th/0008066. 
[5] G. Zs. Tóth, J. Phys. A37 (2004) 9631-9650, hep-th/0406139.

[6] V. P. Yurov and Al. B. Zamolodchikov, Int. J. Mod. Phys. A6 (1991) 4557-4578.

[7] G. Mussardo, V. Riva and G. Sotkov, Nucl. Phys. B687 (2004) 189-219, hepth/0402179.

[8] J. Goldstone and R. Jackiw, Phys. Rev. D11 (1975), 1486-1498.

[9] Al. B. Zamolodchikov, Int. J. Mod. Phys. A10 (1995) 1125-1150.

[10] Z. Bajnok, L.Palla, G. Takács and F. Wágner, Nucl. Phys. B587 (2000) 585-618, hep-th/0004181.

[11] G. Delfino, G. Mussardo and P. Simonetti, Nucl. Phys. B473 (1996) 469-508, hepth/9603011.

[12] G. Feverati, F. Ravanini and G. Takács, Phys. Lett. B430 (1998) 264-273, hepth/9803104.

[13] G. Feverati, F. Ravanini and G. Takács, Nucl. Phys. B540 (1999) 543-586, hepth/9805117.

[14] C. Destri and H.J. De Vega, Nucl. Phys. B438 (1995) 413-454, hep-th/9407117. C. Destri and H.J. de Vega, Nucl. Phys. B504 (1997) 621-664, hep-th/9701107. 